\title{
Research on medical image segmentation based on FCM algorithm
}

\author{
Wang Jinguo ${ }^{1,}$ a , Wang $\mathrm{Na}^{2, b^{*}}$ (corresponding author), Wang rui ${ }^{3, \mathrm{c}}$ \\ ${ }^{1}$ Department of Urology, the First Hospital of Jilin University, China \\ ${ }^{2}$ Department of Anesthesiology, the First Hospital of Jilin University, China \\ ${ }^{3}$ Department of Anesthesiology, the First Hospital of Jilin University, China \\ âwangjinguolily@163.com, ${ }^{\mathrm{b}}$ lilyly12345@163.com, ${ }^{\mathrm{C} X i a o b e n 6666 @ 126 . c o m ~}$
}

Keywords: Medical image. segmentation algorithm. FCM algorithm .

Abstract. This paper summarizes and compares the current medical image segmentation algorithm based on FCM algorithm. Based on the summary of the existing research results, this paper further designs the correlation algorithm, in order to obtain better segmentation results and higher operating efficiency.

\section{Introduction}

Since 1999, Ahmed has improved the FCM algorithm and applied it to the field of image segmentation. There have been many improved FCM algorithms, such as FCMS1 and FCMS2[1][2], which should be used for image segmentation. The algorithms are deeply research on the analysis, the FCM algorithm in medical image segmentation is divided into two categories: Improved FCM algorithm based on neighborhood information, Improved FCM algorithm based on efficiency improvement. Although the FCM algorithm in image segmentation has made great progress in the last 15 years, the correlation algorithm still has a large space for improvement[3].

Along with the rapid development of information technology and the continuous improvement of the level of computer application, the rapid development of medical imaging instrument is promoted. To make full use of medical images provided by these medical imaging instruments, it can help doctors to carry out computer aided diagnosis, make the internal and external operations planning, and analyze the structure and the process of the disease, and improve the accuracy of the diagnosis. The key problem of medical image is the correct and fast segmentation of the image. Over the years, many scholars and researchers have done a lot of research on medical image segmentation, and achieved a lot of results, but to achieve the right, fast segmentation and a long way to go. How to improve the accuracy and speed of medical image segmentation is still the bottleneck of the current recognition[4].

\section{Overview of image segmentation}

In essence, image segmentation is based on the image of the pixels of a feature, for a given image segmentation get the partition. That is to divide the image into several disjoint parts, which meet the following two conditions[5][6]:

$$
\begin{aligned}
& \bigcup_{i=1}^{n} I_{i}=I \\
& I_{i} \mid I_{j}=\Phi \quad i \neq j
\end{aligned}
$$


Compared with general natural images, there are three special phenomena in medical images because of the mechanism of imaging and the reasons of the device itself: partial volume effect(PVE), intensity in-homogeneity(IIH), noise. In general, the phenomenon of partial volume effect is a common problem in medical imaging equipment. It refers to the signal acquisition at the same level, if there are two or more than two kinds of material, the signal is the average value of the material signal, and not a certain type of signal value. The PVE effect is a phenomenon that the gray value of a pixel is the gray value of the pixel. It is not the same as the gray value of the pixel gray value and the gray value of the central pixel. Because of the partial volume effect, the intensity is not the same. Generally speaking, noise in medical images is multiplicative noise[7].

\section{FCM algorithm in image segmentation}

According to the above analysis, because of the existence of partial volume effect, the pixel intensity values are the average values of different density on the same level. Therefore, the intensity of a pixel may be involved in many tissues or organs. Therefore, the traditional k- means algorithm is not suitable for medical image segmentation, because in this algorithm, the pixel can only belong to one cluster, which has nothing to do with other clustering. This process will result in a good segmentation result. Therefore, the medical image segmentation technology based on fuzzy technology is produced, and the most famous one is the fuzzy c- means algorithm(FCM).

FCM algorithm is proposed by Dunn, which is later promoted by Bezdek. It is a classical clustering algorithm. So far, FCM algorithm has been successfully applied in machine learning, pattern recognition, computer vision and other related fields Nan. In essence, the FCM algorithm is to complete the image segmentation by minimizing the weighted distance between the pixels and the clustering center. In FCM algorithm, the weighted distance is referred to as the objective function or the energy function:

$$
J=\sum_{i=1}^{C} \sum_{j=1}^{n} u_{i j}^{m} d_{i j}^{2}
$$

In which, $\mathrm{C}$ is the default clustering number; $\mathrm{n}$ is the number of pixels in the image. $u_{i j}$ is the second part of the $\mathrm{j}$ pixel belonging to the class $\mathrm{i}$ membership degree. It meet the following condition:

$$
0 \leq u_{i j} \leq 1 \quad \text { and } \quad \sum_{i=1}^{C} u_{i j}=1
$$

$\mathrm{m}>1$ is the default fuzzy factor. $d_{i j}=\left\|x_{j}-v_{i}\right\|$ is the Euclidean distance between the first $\mathrm{j}$ pixels to the i cluster center.

By minimizing the objective function in the type (3), the first $\mathrm{j}$ pixels can be obtained under different clustering membership degrees $u_{i j}(i=1,2, \ldots \ldots . C)$. When the image is segmented, the pixel $\mathrm{j}$ is divided into $\mathrm{k}$ class, in which:

$$
k=\arg \max _{i}\left\{u_{i j}, i=1,2, \ldots \ldots, C\right\}
$$

In order to minimize the objective function of the type (3), the constraint conditions are 
considered to be satisfied by the membership degree $\sum_{i=1}^{C} u_{i j}=1$, So the objective function is to minimize the objective function based on the Lagrange operator method:

$$
F=\sum_{i=1}^{C} \sum_{j=1}^{n} u_{i j}^{m} d_{i j}^{2}+\sum_{j=1}^{n} \lambda_{j}\left(\sum_{i=1}^{n} u_{i j}-1\right)
$$

Because $\frac{\partial F}{\partial u_{i j}}=0$, it get :

$u_{i j}=\left(\frac{-\lambda_{j}}{m d_{i j}^{2}}\right)^{\frac{1}{m-1}}=\frac{\left(-\lambda_{j}\right)^{\frac{1}{m-1}}}{\left(m d_{i j}^{2}\right)^{\frac{1}{m-1}}}$

According type(7) and constraint conditions $\sum_{i=1}^{C} u_{i j}=1$, we get

$$
\left(-\lambda_{j}\right)^{\frac{1}{m-1}}=\frac{1}{\sum_{k=1}^{C}\left(\frac{1}{m d_{k j}^{2}}\right)^{\frac{1}{m-1}}}
$$

The type (8) into (7), we get:

$$
u_{i j}=\left[\sum_{k=1}^{C}\left(\frac{d_{i j}^{2}}{d_{k j}^{2}}\right)^{\frac{1}{m-1}}\right]^{-1}
$$

Because $\frac{\partial F}{\partial u_{i j}}=0$, we get :

$$
u_{i}=\frac{\sum_{j=1}^{n} u_{i j}^{m} x_{j}}{\sum_{j=1}^{n} u_{i j}^{m}}
$$

Repeated application (9) and type (10), the clustering center and the pixel of the membership of the update. When the objective function reaches a minimum, the image segmentation can be accomplished by the membership degree $u_{i j}$ of each pixel of the different clusters according to the formula (5).

From the formula (3), it can be seen that the target function of the FCM algorithm is the minimum, and the distance from the center of the clustering center is given a large membership degree, and the distance from the center of the cluster is given a small degree of membership. However, the formula (3) also shows that the target function of the FCM algorithm only considers the gray value of pixels, and does not take into account other information, which leads to the FCM algorithm is sensitive to the noise in the image, can not get the ideal segmentation results.

FCM algorithm is sensitive to the noise in the image, and can't get the ideal segmentation results. 
Because the noise in medical images is carried by the medical devices themselves, how to suppress the noise in the process of medical image segmentation is the key to ensure the quality of the segmentation. In addition, the FCM algorithm is adopted to deal with the iterative method when the objective function is minimized. In the process of iteration, the initial value of the objective function is larger, but the change is not big in most of the iteration process. The efficiency of FCM algorithm is lower, which can not meet the real-time requirements of image segmentation.

\section{References}

[1] Bernard Ghanem,Narendra Ahuja. Dinkelbach NCUT: An Efficient Framework for Solving Normalized Cuts Problems with Priors and Convex Constraints[J]. International Journal of Computer Vision . 2010 (1).

[2] B. V. Cherkassky,A. V. Goldberg. On Implementing the Push-Relabel Method for the Maximum Flow Problem[J]. Algorithmica . 1997 (4).

[3] Vicent Caselles,Francine Catté,Tomeu Coll,Françoise Dibos. A geometric model for active contours in image processing[J]. Numerische Mathematik . 1993 (1).

[4] Geman S,Geman D.Stochastic relaxation, Gibbs distribution and the Bayesian restoration of images. IEEE Transactions on Pattern Analysis and Machine Intelligence . 1984.

[5] Vladimir Kolmogorov,Ramin Zabih. What energy functions can be minimized via graph cuts?. IEEE Transactions on Pattern Analysis and Machine Intelligence . 2004.

[6] Boykov Y,Jolly M P.Interactive Graph Cuts for Optimal Boundary and Region Segmentation of Objects in N-D Images. Proceedings of the International Conference on Computer Vision . 2001.

[7] Greig D,Porteous B,Seheult A.Exact maximum a posteriori estimation for binary images. Journal of the Royal Statistical Society Series B Statistical Methodology . 1989. 